\title{
Androgen receptor-mediated inhibition of cutaneous wound healing
}

\author{
Gillian S. Ashcroft and Stuart J. Mills \\ Cells, Immunology and Development, School of Biological Sciences, University of Manchester, Manchester, United Kingdom
}

Impaired wound healing states in the elderly lead to substantial morbidity, mortality, and a cost to the US Health Services of over $\$ 9$ billion per annum. In addition to intrinsic aging per se causing delayed healing, studies have suggested marked sex-differences in wound repair. We report that castration of male mice results in a striking acceleration of local cutaneous wound healing, and is associated with a reduced inflammatory response and increased hair growth. Using a hairless mouse model, we have demonstrated that testosterone reduction stimulates the healing response not through hair follicle epithelial/mesenchymal cell proliferation, but directly via effects on wound cell populations. We suggest that endogenous testosterone inhibits the cutaneous wound healing response in males and is associated with an enhanced inflammatory response. The mechanisms underlying the observed effects involve a direct upregulation of proinflammatory cytokine expression by macrophages in response to testosterone. Blockade of androgen action systemically, via receptor antagonism, accelerates healing significantly, suggesting a specific target for future therapeutic intervention in impaired wound healing states in elderly males.

J. Clin. Invest. 110:615-624 (2002). doi:10.1172/JCI200215704.

\section{Introduction}

Cutaneous wound healing is a complex process encompassing a number of overlapping events that include leukocyte recruitment, matrix deposition, epithelialization, and ultimately resolution of inflammation with the formation of a mature scar. Impaired agerelated wound healing states - involving both acute wounds that fail to heal and chronic ulcers - are characterized by excessive leukocytosis and subsequently enhanced proteolytic degradation of matrix constituents $(1,2)$. A rapid increase in the elderly population has resulted in a parallel increase in problems associated with age-related delayed wound healing. Treatment of such impaired healing costs the US Health Services over $\$ 9$ billion per year. Additionally, chronic wounds lead to an incalculable degree of suffering, including reduced mobility, wound odor, exudate, and pain. Intriguingly, reports have shown that males heal acute wounds more slowly than females and have an altered inflammatory response (3-5). Epidemiologic studies have not previously reported that sex has an impact on wound repair; however, recent neural network studies have demonstrated that the

Received for publication April 16, 2002, and accepted in revised form June 25, 2002.

Address correspondence to: Gillian S. Ashcroft, Cells, Immunology and Development, School of Biological Sciences, University of Manchester, 3.239 Stopford Building, Oxford Road, Manchester M13 9PT, United Kingdom.

Phone: 011-44-(0)161-275-5673; Fax: 011-44-(0)161-275-3915; E-mail: gillian.s.ashcroft@man.ac.uk.

Conflict of interest: No conflict of interest has been declared. Nonstandard abbreviations used: dehydroepiandrosterone (DHEA); androgen receptor (AR); dihydrotestosterone (DHT); electromobility shift assay (EMSA). male genotype is a strongly positive risk factor for impaired healing in the elderly (5). Despite their potential impact on wound healing, the mechanisms underlying such sex differences have not been elucidated.

One critical mediator of wound healing is the hormone estrogen, which accelerates repair in both human and animal models. Local levels of bioavailable estrogen are altered in the elderly due to a combination of decreased circulating gonadal estrogen (particularly important in postmenopausal females) and markedly reduced levels of the adrenal sex steroid precursor dehydroepiandrosterone (DHEA), resulting in a parallel decrease in androgen and estrogen formation from DHEA aromatization in peripheral tissues (6-8). Estrogen can reverse age-related impaired healing in females when applied topically or given systemically and is associated with reduced local inflammation and enhanced matrix deposition $(4,9)$. In elderly males, the response to estrogen is significantly reduced compared with that in females, suggesting that other, unknown factors are involved beyond the effects of reduced estrogen. One factor that has not been investigated to date is the potential role of androgens in wound repair and local cutaneous inflammation. Elderly males generally maintain testosterone levels, albeit with a gradual reduction with increasing age, and androgens have been reported to be pivotal mediators of local and humoral immune responses in other pathophysiological processes.

In this context, several reports indicate that androgens play a critical role in the immune response and account for differences in outcome based on sex, including susceptibility to sepsis, parasitic infection, and atherosclerosis related to enhanced monocyte adhesion to endothelium (10-12). Androgens have been related to both pro- and anti-inflammatory states at both the sys- 
temic level (13) and the cellular level, modulating IL-1, IL-2, and IL-6 in a variety of cell types including fibroblasts, macrophages (increasing IL-6), Kupffer cells (decreasing IL-6), splenocytes, and osteoblasts (14-17). Moreover, recent in vivo studies have suggested that castration of rats following burn injury significantly reduces systemic levels of proinflammatory TNF- $\alpha$, and that the in vitro macrophage production of IL- 1 and TNF- $\alpha$ is inhibited by androstenetriol $(18,19)$. Taken together, these reports suggest that androgens may exert both anti- and proinflammatory effects that depend on the cell type, animal model, and dose of treatment administered. In this regard, the role of androgens in the cutaneous wound healing response and the effects on local inflammation have not been investigated.

In this study we have demonstrated that castration of male mice results in accelerated cutaneous wound healing and is associated with a dampened inflammatory response and increased matrix deposition. Using a hairless mouse model, we demonstrated that the enhanced hair follicle proliferation resulting from castration was not responsible for such effects on the healing rate. The underlying mechanisms involve a direct effect of testosterone on murine macrophage TNF- $\alpha$ production via the androgen receptor $(\mathrm{AR})$ in parallel to the in vivo downregulation of TNF- $\alpha$ following castration or AR antagonism. Intriguingly, AR blockade accelerates healing in a similar fashion to castration, suggesting a future target for therapeutic intervention to accelerate healing in elderly males.

\section{Methods}

Wound healing experiments. Male C57BL/6 or $\mathrm{br} / \mathrm{hr}$ male wild-type and null mice (Harlan Laboratories Ltd., Indianapolis, Indiana, USA), 8-12 weeks old, were anesthetized with inhaled isofluorane, and the dorsum was shaved and cleaned with alcohol. Ten-weekold male mice that had undergone castration or sham castration 1 month previously were also used for the wound healing experiments. Two equidistant $1-\mathrm{cm}$ full-thickness incisional wounds were made through the skin and panniculus carnosus muscle and were left to heal by secondary intention. Wounds were harvested on days 1, 3, 5, 7, 14, and 21 after wounding and were bisected for histology and for RNA analysis/protein extraction, and immediately snap frozen in liquid nitrogen for RNA analysis/protein extraction. A subgroup of intact C57BL/6 male mice were treated with oral flutamide $(200 \mathrm{mg} / \mathrm{kg} /$ day flutamide in $0.5 \%$ methylcellulose) for 5 days prior to wounding, and day 3 wounds were excised (20). Serum from cardiac puncture of all mice was used to determine circulating testosterone levels using an ELISA kit (ICN, Basingstoke, United Kingdom).

Human wound bealing study. Eighteen health status-defined elderly males (mean age 71 years, $\mathrm{SD}=8.6$ ) underwent two 4-mm punch biopsies, and the wounds were measured by planimetry on day 7 after wounding, as previously described (4). Mean total testosterone levels were measured by standard radioimmunoassay; the normal laboratory range of testosterone is $10-30$ $\mathrm{ng} / \mathrm{ml}$. Sex hormone binding globulin levels did not differ significantly within the cohort. As part of an ongoing study, young (20-39 years) and elderly (60-85 years) males and females (five subjects per group) from a health-status panel underwent two $4-\mathrm{mm}$ punch biopsies from the upper inner arm, and the wounds were excised on day 3,7 , or 14 after wounding (1).

Histology, immunocytochemistry, and image analysis. Histological sections were prepared from male murine and human wound tissue fixed in $10 \%$ buffered formalin and embedded in paraffin. Five-micrometer sections were stained with hematoxylin and eosin or with picrosirius red (for collagen determination) (21), or were subjected to immunohistochemistry with specific antibodies. Antibodies used were N-20 (22), raised against a synthetic peptide localizing to the N-terminal of the human AR (Santa Cruz Biotechnology Inc., Santa Cruz, California, USA), diluted to $1: 10$, with mouse prostate gland used as positive control; Mac-3 (rat anti-Mac-3; Pharmingen, San Diego, California, USA); collagen VII (mouse to human; Chemicon International, Temecula, California, USA); and TNF- $\alpha$ (rabbit polyclonal; Genzyme Pharmaceuticals, Cambridge, Massachusetts, USA), used at a dilution of 1:20 in PBS. Primary antibody was detected using either a TRITC- or FITC-labeled secondary antibody (1:40 dilution for 20 minutes) or using a peroxidase kit (Vector Laboratories Inc., Burlingame, California, USA). Controls using IgG isotype serum were in all cases negative. Image analysis and quantification of cell number per unit area $\left(\mathrm{mm}^{2}\right)$ and wound area (measured below the clot and above the panniculus muscle) were performed using the ImagePro Plus program (MediaCybernetics Inc., Silver Springs, Maryland, USA) as previously described (21). Androgen effects on monocytes. Peritoneal macrophages were isolated from male wild-type C57BL/ 6 mice by intraperitoneal lavage with ice-cold sterile PBS and pooled for subsequent studies. Cells were used at $10^{7}$ cells $/ \mathrm{ml}$ with $1 \mathrm{ml}$ per tube in serum-free phenol red-free medium with or without LPS $(1 \mu \mathrm{g} / \mathrm{ml}$; SigmaAldrich, St. Louis, Missouri, USA), testosterone $\left(10^{-8} \mathrm{M}\right.$; Sigma-Aldrich), $5 \alpha$-dihydrotestosterone ( $5 \alpha$-DHT) $\left(10^{-8} \mathrm{M}\right.$; Sigma-Aldrich), flutamide $\left(10^{-6} \mathrm{M}\right.$; SigmaAldrich), and the estrogen receptor inhibitor ICI $182780(100 \mathrm{nM})$ for 12 hours. RNA was subsequently extracted as described below.

Chemotaxis of monocytes was stimulated in a 12-well chemotaxis chamber (Transwell Plate; Corning-Costar Corp., Acton, Massachusetts, USA), with each well containing $400 \mu \mathrm{l}$ of control media or TGF- $\beta 1(1 \mathrm{pg} / \mathrm{ml})$, testosterone $\left(10^{-8} \mathrm{M}\right)$, or both TGF- $\beta 1$ and testosterone. Prior to the assay, cells were treated with or without testosterone for 12 hours $\left(10^{-6}\right.$ to $\left.10^{-10} \mathrm{M}\right)$. Monocytes were resuspended in $100 \mu \mathrm{l}$ chemotaxis buffer $(1 \times$ Hank's buffer with $0.5 \% \mathrm{BSA})$ in the upper chamber at a final concentration of $3 \times 10^{5}$ per $100 \mu \mathrm{l}$ 
and incubated for 90 minutes at $37^{\circ} \mathrm{C}$ in a humidified atmosphere of $5 \% \mathrm{CO}_{2}$. Cells that migrated across the membrane (pore size, $3 \mu \mathrm{m}$ ) were fixed in $40 \mu \mathrm{l} \mathrm{chemo-}$ taxis fixative (100 mM EDTA and 10\% formaldehyde in PBS) and counted using a hemocytometer.

$R T-P C R, R N a s e$ protection, and hydroxyproline assays. Total RNA was isolated from frozen wound tissue and peritoneal macrophages using Trizol. For PCR, total RNA extracted from wound tissue as above was reverse transcribed and subjected to PCR using primer pairs for murine AR (23), TNF- $\alpha$, and HPRT (housekeeping gene). The sequences were: HPRT, $5^{\prime}$-ACTCTGCTTCAGATCCCTGC and $5^{\prime}$-GGACCAGCAACTTGAAGAGG (266bp product); TNF- $\alpha, 5^{\prime}$-TCCGCTTCTCCGCTGCCA and $5^{\prime}$-CACCTTTGTGTCTGGGACCT (872-bp product); and $A R, 5^{\prime}$-AGTCATCCCTGCTTCATAAC and 5'-ATCCTGGTGGAGTTGTGAAC (394-bp product). Five micrograms of RNA was subjected to RNase protection assays using the mck $3 \mathrm{~b}$ template per manufacturer's instructions (Pharmingen, San Diego, California, USA) (21). As an indication of total collagen content, hydroxyproline levels were determined in wound tissue as previously described (4).

Electromobility shift assay and Western blotting. Nuclear proteins were isolated from wound tissue by homogenization in lysis buffer $(20 \mathrm{mM}$ Tris at $\mathrm{pH} 7.6,120 \mathrm{mM}$ $\mathrm{NaCl}, 1 \% \mathrm{NP}-40,10 \%$ glycerol, $10 \mathrm{mM}$ sodium pyrophosphate, $100 \mathrm{mM} \mathrm{NaF}, 2 \mathrm{mM}$ sodium orthovanadate, $1 \mathrm{mM}$ AEBSF, and $5 \mu \mathrm{g} / \mathrm{ml}$ leupeptin). Electromobility shift assay (EMSA) for NF- $\mathrm{KB}$ was performed using a radiolabeled NF- $\kappa B$ consensus oligonucleotide probe (Promega Corp., Madison, Wisconsin, USA) as previously described (24).

For Western blotting, protein was extracted from murine wound tissue and normal skin using a detergent buffer, and $10 \mu \mathrm{g}$ protein was used for the blot as previously described (1). Rabbit anti-TNF- $\alpha$ (Genzyme Pharmaceuticals) or rabbit anti-AR antibody (Santa Cruz Biotechnology Inc.) was used at $2 \mu \mathrm{g} / \mathrm{ml}$ in an overnight incubation, followed by detection using the AP kit per the manufacturer's instructions (Bio-Rad Laboratories Inc., Herts, United Kingdom). Fresh mouse prostate tissue was used as a positive control for AR immunoblotting.

Statistical analysis. Statistical differences were determined using the Student $t$ test or multivariate ANOVA for parametric analyses and linear regression, and by Mann-Whitney U test for nonparametric analysis. All data represent mean $\pm \mathrm{SD}$. A $P$ value less than 0.05 was considered significant.

\section{Results}

AR expression during normal wound healing. Assessment of the temporal profile and cellular sources of AR expression during normal wound healing in wild-type C57BL/6 mice following full-thickness incisional wounds excised on days 1-21 after wounding revealed a distinct pattern of expression. Scattered basal epidermal cell and hair follicle staining was apparent in normal skin (Figure 1a), corroborating previous studies in rat and primate skin (25). Intact skin from castrated male mice showed no differences in AR staining
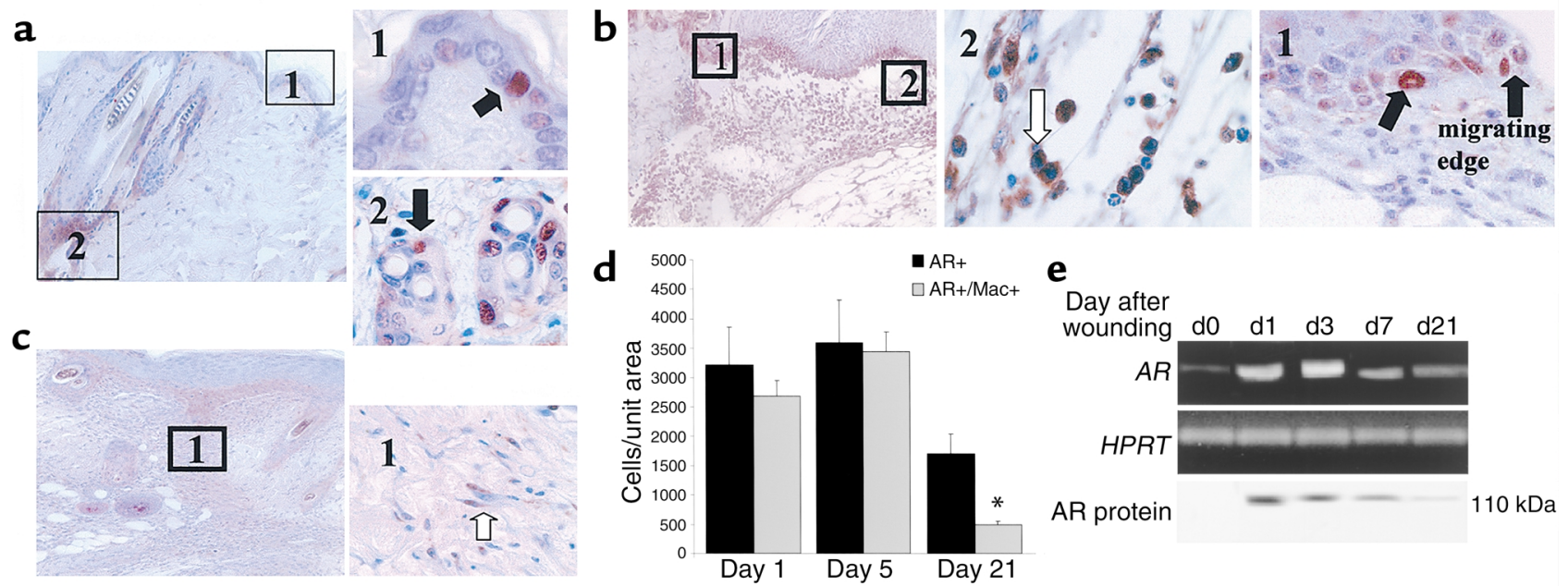

\section{Figure 1}

AR localizes to keratinocytes, inflammatory cells, and fibroblasts during wound healing. (a) Normal skin immunostaining for AR (left panel) at low magnification $(\times 20)$ illustrates epidermal and hair follicle staining. Right panels show high magnification $(\times 100)$ of basal epidermal cells (area 1) and hair follicles (area 2). (b) Day 3 wounds: left panel is a low-magnification image of AR-stained tissue. Area 2 is a highmagnification image of macrophages, and area 1 shows basal and suprabasal epithelial staining at the migrating edge. (c) A day-14 wound (left panel, low magnification) with cells morphologically resembling fibroblasts staining positively for AR; (area 1, right panel) is a highmagnification image. Images are representative of ten wounds stained per timepoint from male mice. (d) AR-positive cells were quantified from day 1 to day 21 after wounding; those colocalizing with Mac-3 were quantified separately. (e) RT-PCR for AR and the housekeeping gene HPRT in wound tissue showed a temporal increase in expression through day 3 , with a decrease by day 21 . d0, normal, unwounded skin. Bottom panel shows a Western blot for $A R(110 \mathrm{kDa})$ demonstrating an increase in protein levels from basal ( $\mathrm{d} 0)$ to day 7 , then a decline in protein to day 21 after wounding. Blot is representative of three experiments using five mice per timepoint. 
$\mathbf{a}$
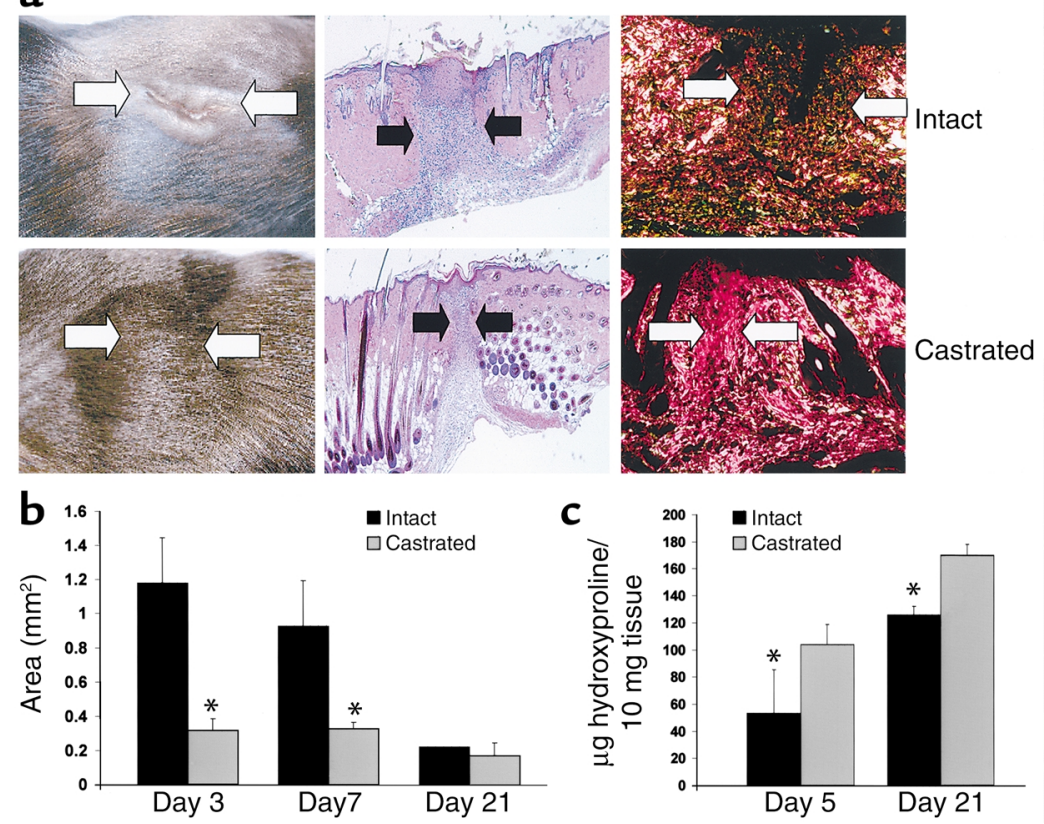

\section{Figure 2}

Accelerated healing in the absence of male gonadal steroids. (a) Left and central panels show macroscopic and histological day 5 wounds illustrating the smaller (healed) wounds in castrated animals compared with intact animals. Collagen content was markedly increased in the wounds of the castrated animals at day 5 (right panels). Magnification: $\times 10$ (center, hematoxylin and eosin); $\times 20$ (right, picrosirius red). (b) Cross-sectional wound areas were significantly reduced on days 3 and 7 after wounding in the castrated animals $(n=6-8$ per group; $\left.{ }^{*} P<0.05\right)$. (c) Hydroxyproline levels in wound tissue were significantly increased in the castrated mice compared with intact mice at days 5 and 21 after wounding. Extraction and analysis were performed on individual wounds from five mice per group at day 5 , and on five samples of pooled wounds (three wounds per tube) at day 21 after wounding. Data represent mean value per 10 mg tissue. ${ }^{*} P<0.05$. patterns compared with uncastrated animals (data not shown). Increased numbers of basal and suprabasal epidermal cells stained positively in the migrating wound epithelium from day 3 after wounding (Figure 1b). By day 2, staining of the wound epithelium resembled that of adjacent normal skin, with intermittent basal keratinocyte staining, possibly reflecting a subset of androgen-responsive cells. Inflammatory cell staining was also evident from day 3 through day 14 after wounding (Figure $1 \mathrm{~b}$ ), with a marked decrease in inflammatory cell numbers by day 21 after wounding. In addition, scattered cells morphologically resembling fibroblasts stained positively for AR beginning on day 7 after wounding (Figure 1c, day 14) and per- sisting until day 21. Quantitation of dermal AR-positive cells and cells positive for both AR and Mac-3 (Figure 1d) demonstrated an increase in AR-positive cells from day 1 to day 5 after wounding; the majority of these cells were macrophages (dual stained). After day 5, AR-positive cells declined at the wound site, and an increase in AR-positive, Mac-3-negative cells was observed, reflecting an influx of AR-positive fibroblasts. Wound tissue mRNA and protein levels of the AR were increased strikingly on days 1 and 3 after wounding and decreased thereafter to day 21 , reflecting the immunostaining pattern (Figure 1e). The spatial localization of AR expression during early wound healing, associated with epithelialization and in-

\section{Figure 3}

Increased hair follicle proliferation following gonadectomy does not contribute to the wound healing response. (a) Day 3 macroscopic and histological wounds from intact wild-type and intact null $(h r / h r)$ mice showed no differences in healing. By contrast, castrated $h r / h r$ and castrated wild-type mice healed significantly more quickly. Magnification: $\times 10$. (b) Quantification of wound areas at day 3 illustrated a significant decrease in area (accelerated healing) in the castrated $h r / h r$ null (KO) animals compared with the intact $h r / h r$ littermates, and wild-type (WT) castrated compared with wild-type intact. $n=6$ per group; ${ }^{*} P<0.05$.

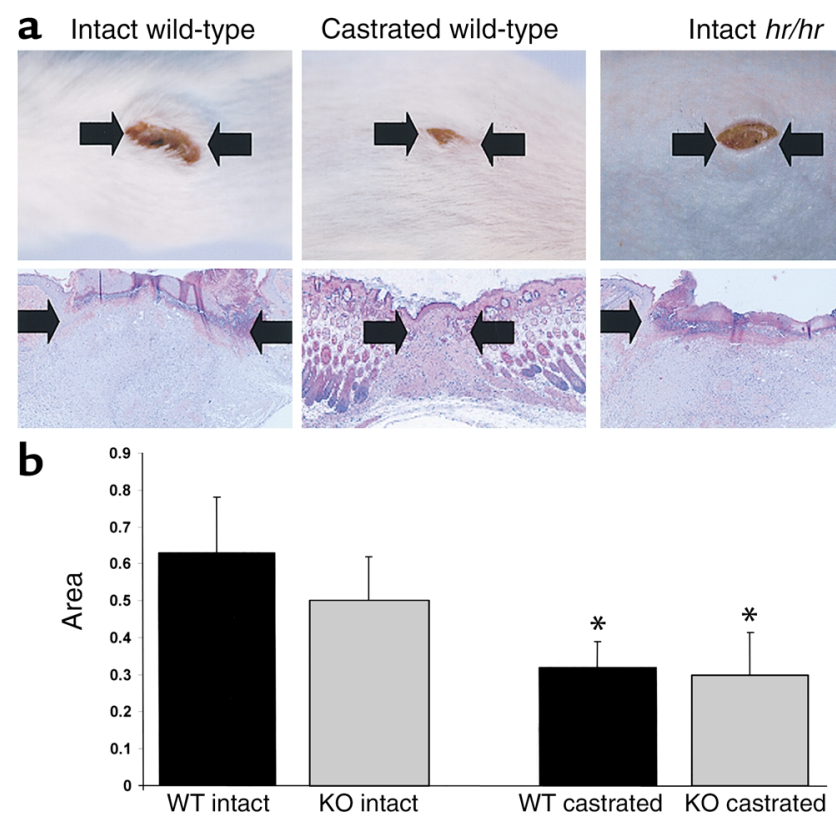


creased cellular infiltrate, implicated a role for this receptor in inflammation and/ or repair.

Castration accelerates wound healing in vivo. Impaired age-related wound healing states, involving both acute wounds that fail to heal and chronic ulcers, are characterized by excessive leukocytosis and subsequently enhanced proteolytic degradation of matrix constituents $(2,3)$. Elderly males heal more slowly than elderly females, with reduced matrix deposition and an altered local inflammatory response; the mechanisms underlying such sex differences are unknown. In order to determine the role of male gonadal hormones in this response, we rendered C57BL/6 mice hypogonadal by castration (with parallel sham-castrated and intact control groups), and 1 month later mice were subjected to two $1-\mathrm{cm}$ incisional full-thickness dorsal woundings after shaving. Wound healing was significantly accelerated in the castrated mice compared with the intact animals, with reduced wound areas assessed both macroscopically and microscopically (Figure 2, a and b). Quantification of cross-sectional areas indicated a significant reduction in area at days 3 and 7 after wounding in the castrated animals (Figure $2 \mathrm{~b}$ ). Shamoperated mice showed no healing differences compared with intact mice (data not shown). Collagen I matrix deposition as assessed histochemically (Figure 2a, picrosirius red staining with collagen fibers appearing red) was markedly increased in the wounds of the castrated animals. In accordance with this, hydroxyproline levels were significantly increased in microdissected wound tissue from castrated mice compared with intact mice at days 5 and 21 after wounding (Figure 2c).

Role of hair follicle proliferation in wound healing. Recently both testosterone and estradiol have been implicated as inhibitors of hair growth in a murine model (26). Since hair follicle dermal and epidermal cells may contribute to a rapid rate of repair, we investigated whether the acceleration of healing in the absence of male gonadal steroids was secondary to the remarkable stimulation of hair growth observed in the castrated mice. (27). We used 12-week-old hairless $(b r)$ mice that carry a mutation at the hairless locus leading to hair follicle degeneration into utriculi and dermal cysts shortly after birth (28). The loss of a functional $b r$ gene product (a putative zinc finger transcription factor) results in destruction of the normal hair follicle architecture and increased apoptosis of dermal papilla cells. Wounds were excised at day 3 or day 7 after wounding in null mice and age-matched wild-type littermates. Intriguingly, no differences were observed in the rate of healing (wound area) between the intact wild-type and intact $b r / b r$ genotypes (Figure 3), bringing into question the putative role of dermal
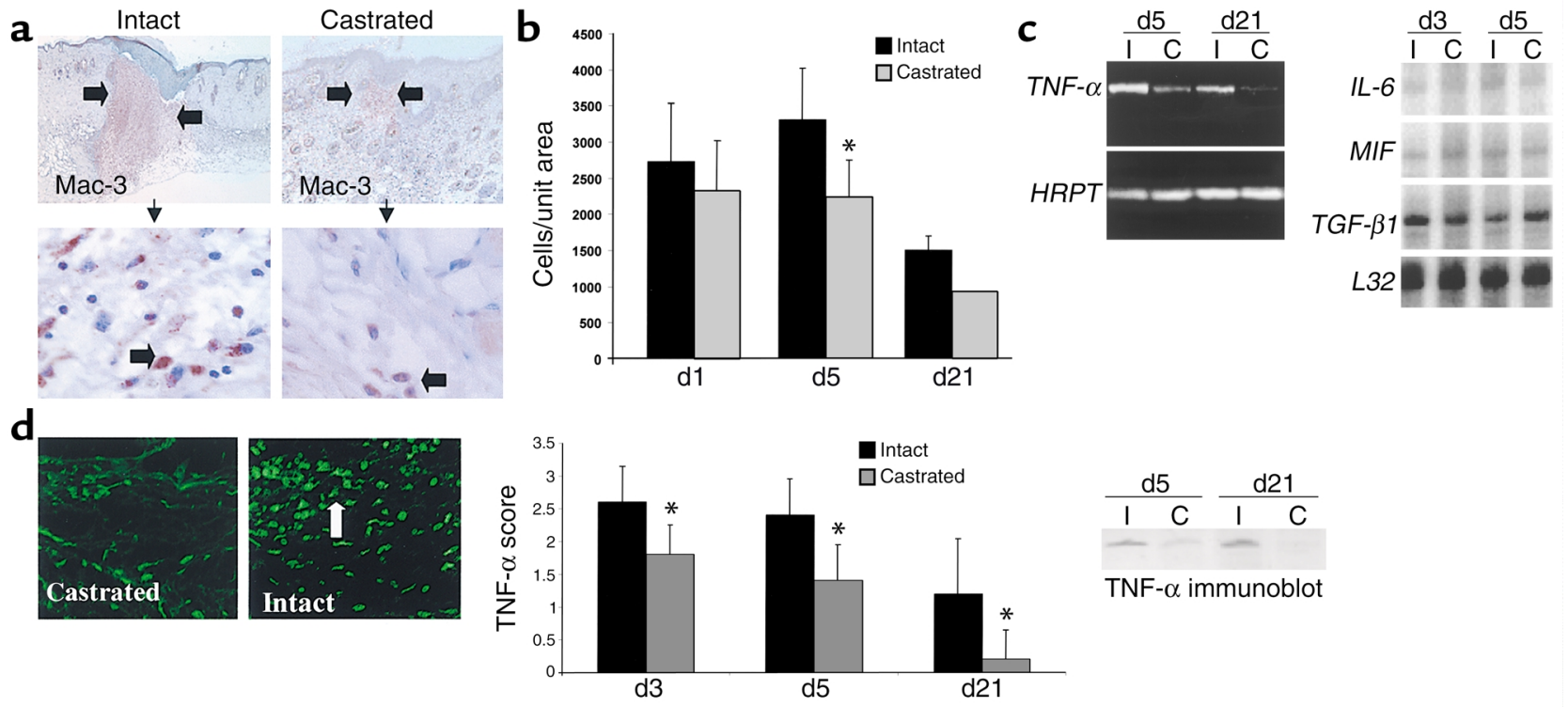

\section{Figure 4}

Castration results in a dampened local inflammatory response and reduced expression of proinflammatory TNF- $\alpha$. (a) Upper panels are low-magnification $(\times 20)$ images of inflammatory cell staining for Mac-3. Mac-3-positive cells (arrow, bottom panels) were increased in day 3 wounds of intact mice compared with their castrated littermates (magnification: $\times 100$ ). (b) Quantification of Mac-3-positive cells per unit area $\mathrm{mm}^{2}$ showed a significant increase in the intact animals at day 5 after wounding compared with castrated mice. (c) Expression of TNF- $\alpha$ was reduced at days 5 and 21 in the wounds of the castrated mice (C) compared with wounds of intact mice (I). Wound tissue was pooled from five mice, and the gel shown is representative of three experiments. Right panel illustrates a representative RNase protection assay (of three replicate experiments) showing no differences in tissue expression of IL-6, macrophage migration inhibitory factor (MIF), or TGF- $\beta 1$ between intact and castrated mice. (d) TNF- $\alpha$ protein levels were increased in wounds of intact compared with castrated mice as illustrated by day 5 immunostaining (left panels, representative of six wounds stained per group). Quantification of immunostaining (graph) showed a significant increase at days 3, 5, and 21 in the wounds of the intact mice compared with the castrated mice. $n=6$ per timepoint. Western blot analysis of wound tissue showed increased levels of TNF- $\alpha$ at days 5 and 21 in wounds of intact mice compared with those of castrated mice. Blot is representative of three experiments using five mice per group. ${ }^{*} P<0.05$. 

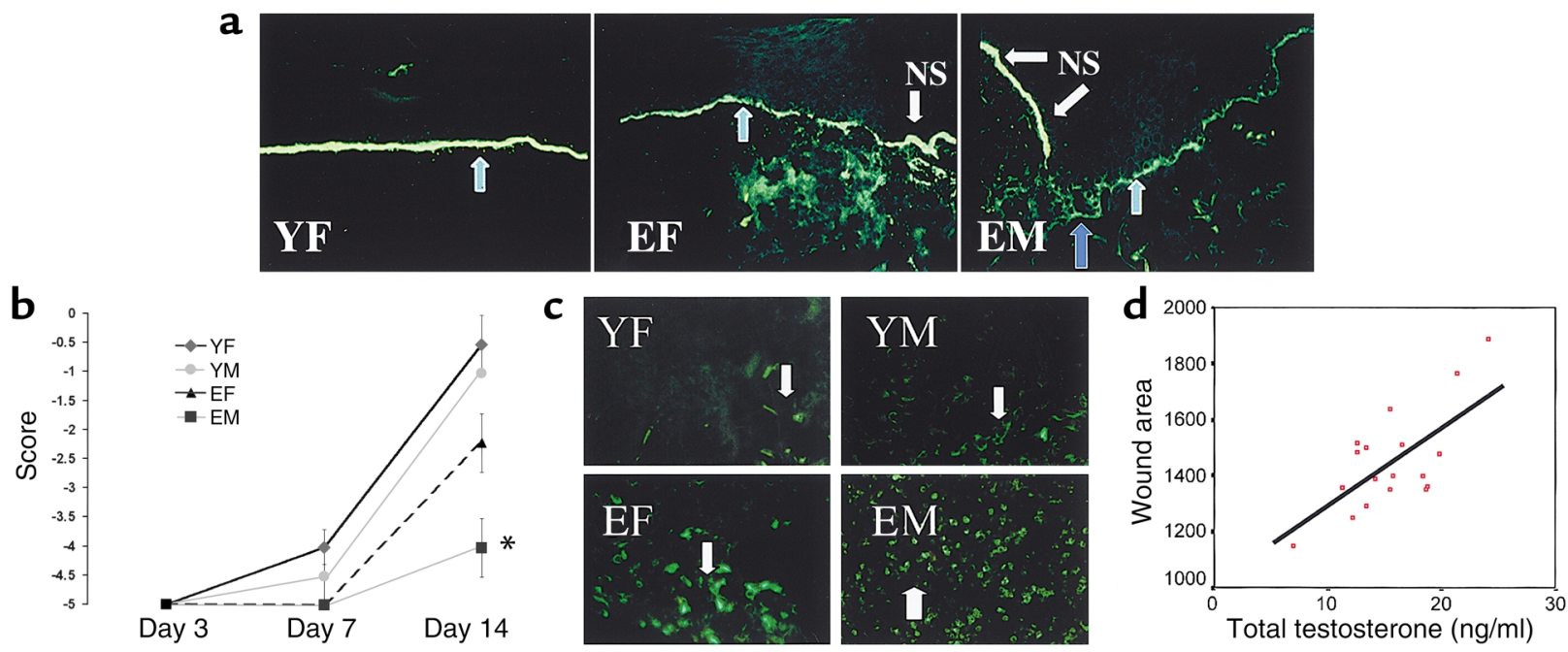

Figure 5

Sex differences in human wound healing. (a) Collagen VII immunostaining at day 14 after wounding illustrates complete reformation of the basement membrane in the young female (YF, left, arrow), whereas staining in the elderly female (EF) is patchy and punctate (green arrow), and is significantly fainter in the elderly male (EM, green arrow). NS, normal skin adjacent to wound. Young males healed similarly to young females, thus only the young female section is shown. (b) Quantification of collagen VII immunostaining where $0=$ normal (unwounded) skin intensity. A significant $\left({ }^{*} P<0.05\right)$ decrease in staining was apparent at day 14 in the elderly males. (c) TNF- $\alpha$ immunostaining at day 7 increased markedly with age, and dramatically in the elderly male. YM, young male. Arrows indicate positively staining macrophages. (d) Systemic testosterone levels in elderly health status-defined human males strongly correlates with impaired healing of acute wounds (increased wound area at day $7 ; P=0.001 ; r^{2}=0.459$ ).

sheath cells in the normal physiological wound healing response (27). To determine the role of hair follicle activity in wound healing in the absence of testosterone, we carried out parallel wounding studies in castrated and intact $b r$ null mice. In agreement with our previous studies, castration resulted in accelerated healing, suggesting that testosterone inhibited healing via a direct mechanism involving the epidermal/dermal cell populations or recruitment of the local inflammatory response. In addition, no differences in the rate of healing were observed between wild-type castrated and null castrated mice (Figure 3, a and b).

Effects of androgens on inflammatory mediators. Accumulating evidence implicates inflammation as a causative factor in delayed healing because it leads to proteolytic destruction of collagen and fibronectin, and suggests that the inflammatory response is inappropriately excessive in the absence of infection $(2,3)$. Significantly, the local inflammatory response was dampened in the wounds of castrated animals at day 3 after wounding. Specifically, Mac-3-positive cells were reduced in number in the wounds of the castrated mice (Figure 4, a and b). In a number of pathophysiological tissue processes, increased inflammation can be attributed to altered TNF- $\alpha$ levels (29). We investigated this response by performing PCR on RNA extracted from wound tissue using primers for TNF- $\alpha$ and the housekeeping gene HPRT. In wounds from both castrated and intact mice, TNF- $\alpha$ was first observed on day 3, was maximal at days 3-5, and decreasing through day 21(Figure 4c). Reduced expression of the proinflammatory cytokine TNF- $\alpha$ was observed on days 5 and 21 after wounding in castrated mice compared with intact animals (Figure 4c). This was a TNFspecific effect, as demonstrated by the lack of effect on expression levels of other cytokines/chemokines following castration, including TGF- $\beta 1$, IL- 6 , and macrophage migration inhibitory factor (Figure 4c). The decrease in TNF- $\alpha$ expression was paralleled by a reduction in protein levels as determined by quantitation of immunostaining (Figure $4 \mathrm{~d}$, graph and representative day 5 immunostaining) and by immunoblotting at days 5 and 21 after wounding (Figure $4 d$ ). These data implicated TNF- $\alpha$ as an inducible factor in the local wound healing response that is regulated directly or indirectly by gonadal steroids.

Sex differences in buman wound healing. In order to determine whether these hormonal effects were relevant in human wound healing, we investigated the differences in healing between sexes in healthy human subjects. We have previously reported that elderly males heal more slowly than elderly females, with reduced matrix collagen deposition (4). By analysis of acute wound tissue from health status-defined subjects, we have shown that collagen VII deposition is also dramatically reduced with increasing age (Figure 5 , a and b), particularly in elderly males. In concordance with our animal studies, wound TNF- $\alpha$ levels were shown to be increased with age on day 7 after wounding, with a marked increase in macrophages staining positively in the wounds of elderly males (Figure $5 c$ ). Whereas sustained systemic and local estrogen levels in both young females and males may contribute to reduced inflammation via direct effects on cell 
adhesion molecule expression (4), the absence of such protective anti-inflammatory action in the elderly corresponds to increased inflammation and TNF- $\alpha$ expression. Moreover, in elderly males, reduced estrogen coupled with maintained levels of bioactive testosterone results in a failure to dampen the TNF- $\alpha$ response (Figure $5 \mathrm{c}$ ). Corroborative evidence for an inhibitory role for testosterone in the healing response came from an investigation into the relationship between wound area and systemic testosterone levels. In elderly males, there was a significant delay in repair with increasing testosterone levels (Figure $5 \mathrm{~d}$ ), strongly implicating this hormone as a direct or indirect modulator of age-related wound repair.

AR blockade accelerates healing in males. Since differences in wound healing between sexes appeared to be significant in humans, we further investigated the mechanisms underlying these responses in an animal model. Male gonads secrete a number of factors that may influence wound repair. In addition to the predominant hormone testosterone and small quantities of estrogen, other factors produced by the testes have been shown to modulate wound repair in animal models, including activin and follistatin (30-32). We reasoned that since testosterone is produced in the most significant quantities, coupled with the correlation in humans between delayed healing and testosterone levels, the effects we observed after castration were modulated by gonadal androgens. To determine the mechanisms underlying the effects of castration, we used AR blockade with oral administration of flutamide prior to and during the wound healing process. Our results suggest that flutamide treatment results in accelerated healing and dampened inflammation, mimicking the effects of castration (Figure 6, a and b), and was associated with reduced tissue expression of TNF- $\alpha$ (Figure $6 c)$. Recent reports suggest that both gonadal androgens and adrenal sex steroid precursors in primates may act not only through the AR but also via the estrogen receptor. Our findings are in keeping with gonadal androgens acting specifically through the AR to reduce local tissue inflammation and modulate wound repair. Since TNF- $\alpha$ activates NF- $\kappa B$, which in turn induces gene expression of a plethora of proinflammatory cytokines, including TNF- $\alpha$ itself, we investigated the activity of NF- $\mathrm{KB}$ in wound tissue nuclear extracts from control and flutamide-treated male mice. EMSA showed NF-KB activity to be strongly increased in day 3 wound tissue compared with normal skin; activation was suppressed by flutamide treatment (Figure 6c).
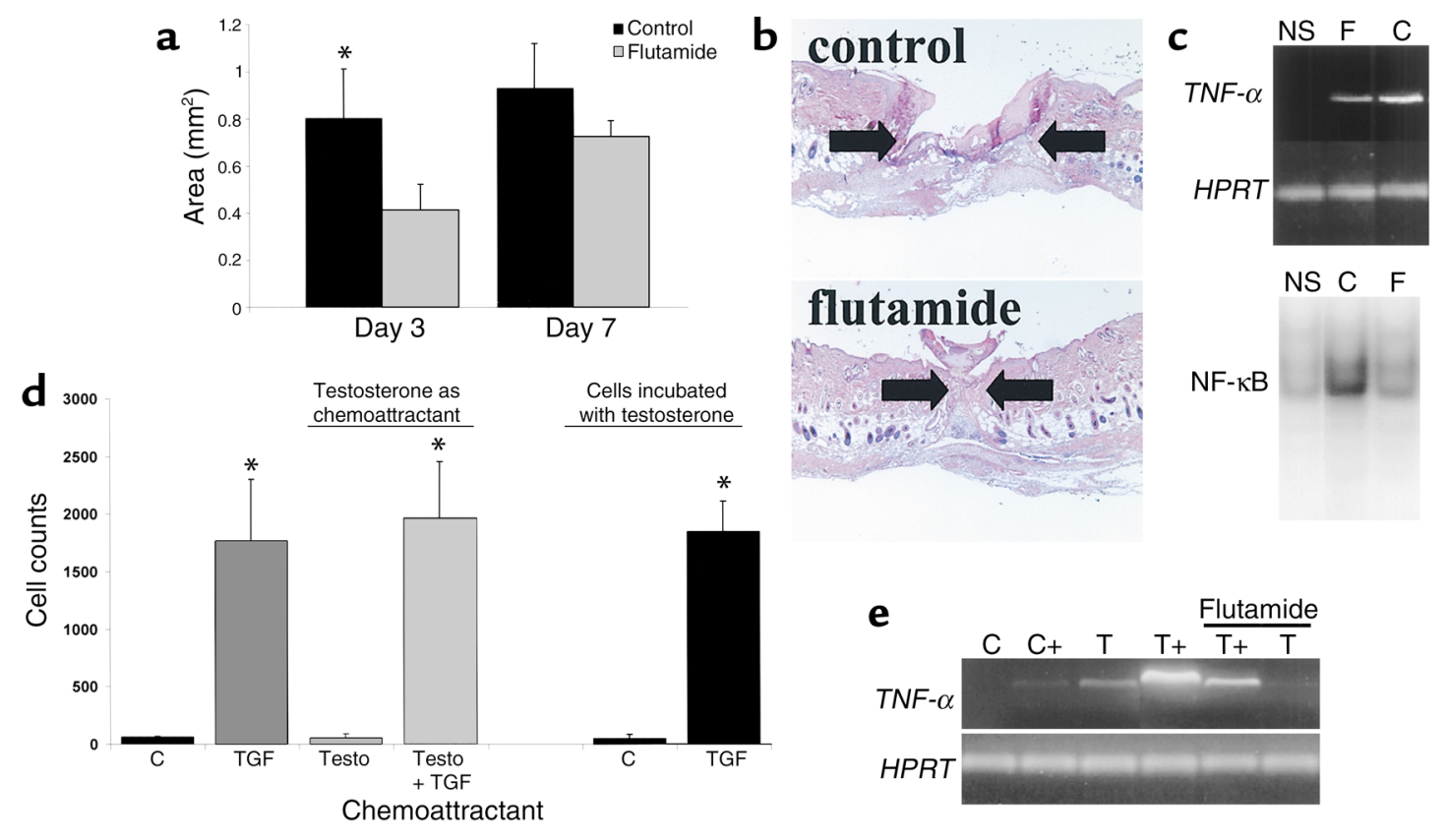

\section{Figure 6}

AR blockade accelerates healing and directly inhibits macrophage TNF- $\alpha$ expression. (a) Flutamide treatment significantly accelerates healing, as illustrated by reduced wound cross-sectional areas at day 3 after wounding $\left({ }^{*} P<0.05\right)$ and by histological analysis (b) of day 3 wounds (arrows demarcate wound edge). (c) Reduced TNF- $\alpha$ expression at day 3 after wounding following flutamide treatment ( $F$, oral flutamide; C, vehicle control; NS, normal skin from control animal). HPRT was used as the housekeeping gene. Bottom panel represents EMSA illustrating increased NF-KB binding activity in day 3 wounds (control) compared with normal skin; binding is markedly reduced in the day 3 wounds of flutamide-treated animals. (d) TGF- $\beta 1$ (TGF) acted as a potent chemoattractant for murine peritoneal macrophages (C, control medium; $\left.{ }^{*} P<0.05\right)$. Testosterone (Testo) had no effect on chemotaxis when used in conjunction with TGF- $\beta 1$ or alone as a chemoattractant, nor when the cells were pretreated with testosterone. (e) LPS-induced TNF- $\alpha$ mRNA expression by murine macrophages. C, control; +, LPS. Testosterone markedly increased TNF- $\alpha$ expression both basally ( $T$ ) and when cells were LPS-activated (T+). Flutamide treatment significantly reduced testosterone-induced TNF- $\alpha$ expression levels. Wound tissue was pooled from four mice, and the gel shown is representative of three experiments. 
Such data suggest a potential role for the AR in mediating the positive feedback loop that exists between TNF- $\alpha$ and NF- $\kappa B$ activity.

To determine the specific cellular and molecular mechanisms whereby androgens modulate the inflammatory profile, with downstream effects on matrix deposition and healing rates, we investigated the androgenmediated responses of murine peritoneal macrophages, focusing on in vitro chemotactic effects and cytokine expression. Whereas TGF- $\beta 1$ acted as a potent chemoattractant for macrophages, testosterone had no effect on migration, either as a chemoattractant in its own right or acting synergistically with TGF- $\beta 1$ (Figure $6 \mathrm{~d}$ ). Moreover, treatment of macrophages with testosterone had no effect on the subsequent migratory response toward medium alone or toward TGF- $\beta 1$, suggesting that testosterone failed to induce morphological/physical perturbations required for cell transit. Increasing doses of $5 \alpha$-DHT similarly showed no effects (data not shown). Intriguingly, testosterone upregulated murine macrophage TNF- $\alpha$ expression above and beyond LPS induction (Figure 6e). 17 $\beta$-estradiol in a dose-response study had no effect on TNF- $\alpha$ levels (data not shown). Flutamide markedly inhibited androgen-induced upregulation, both in the basal state and following LPSinduced activation (Figure 6e); however, estrogen receptor inhibition with ICI 182780 had no effect at any concentration (data not shown). These data strongly implicate the AR as the pathway through which testosterone directly upregulates TNF- $\alpha$ expression and modulates the inflammatory response in vivo.

\section{Discussion}

Testosterone is the principal circulating androgenic hormone secreted by the testes of mature male mammals. Levels gradually decline with increasing age, but the hormone remains significantly bioactive throughout the life span. In male primates after puberty, more than $95 \%$ of circulating testosterone is derived from testicular secretion, with the remainder arising from metabolic conversion of precursors that are predominantly secreted by the adrenal cortex, such as DHEA, DHEA sulfate, and androstenedione. These weak androgens constitute a reservoir of precursors for conversion peripherally to bioactive testosterone. In the skin, adrenal precursors and testosterone itself can be aromatized to estrogen, with subsequent liganddependent activation of the estrogen receptor. In aging males, adrenal production of sex steroid precursors falls dramatically, with reduced levels of gonadal estrogen and altered levels of extragonadal steroid aromatization to tissue estrogen. We have previously documented a pivotal role for estrogen in accelerating healing in both animal models and elderly humans. However, the reduced response to exogenous estrogen in males compared with females, coupled with the observation that elderly males heal more slowly than females, suggested alternative inhibitory mechanisms in males beyond the effects of reduced estrogen.
Despite a documented role of testosterone as an immunoregulator with organ-specific effects, a potential endogenous role in cutaneous wound healing has never previously been reported.

Skin is one organ classified as an androgen-sensitive tissue. It expresses the two genes encoding $5 \alpha$-reductase, which converts testosterone to its more active metabolite, $5 \alpha$-DHT; both enzymes act through the cell-specific AR. The 90-kb AR is a ligand-activated transcriptional factor (the gene for which was cloned in 1988) that encodes three major functional domains: a modulatory N-terminal domain, a DNA-binding domain, and a C-terminal androgen-binding domain $(33,34)$. Expression of AR is inducible by testosterone and $5 \alpha$-DHT in SaOS- 2 and US-OS cell lines, mediating a positive feedback loop (35). We report that AR is expressed by a variety of cell types not only within normal skin but also in acute wounds, including epithelial cells, hair follicles, fibroblasts, and macrophages. Moreover, the spatial and temporal localization of AR in the skin does not change with age in human males (G.S. Ashcroft, unpublished data). Here we demonstrate that castration or direct AR blockade accelerates wound healing through a dampened inflammatory response, increased matrix deposition, and downregulation of TNF- $\alpha$. These effects of androgens on the wound healing process and the parameters assessed suggest that androgens modulate multiple pathways that involve the local inflammatory response.

Since castration resulted in a striking degree of hair growth after shaving and wounding, we determined the role of hair follicle responses to wound repair in an animal model lacking functional hair follicles. Recent reports suggest that follicular epidermal and mesenchymal cells in normal uninjured skin may contribute to adjacent wound epithelialization and granulation tissue production. However, the dogma that hair follicle activity plays a role in the wound healing response is largely unsubstantiated and has never been formally tested in vivo (27). We demonstrated that intact hairless mice, in which an absence of the $h r$ gene product leads to destruction of the follicular architecture and loss of dermal papilla cells, healed in a similar fashion to age-matched wild-type intact mice. These data suggest that in this model, hair follicle contribution to dermal fibroblast and epithelial cell repopulation is not critical to normal repair. Moreover, the effects of castration appeared to be distinct from increased hair follicle activity in the absence of testosterone, since castrated $\mathrm{hr} / \mathrm{hr}$ null mice also healed more quickly than intact null animals did, but healed similarly to castrated wild-type mice. Such data strongly implicated male gonadal steroids as modulators of wound repair via direct actions on the recruitment/responses of specific cells types infiltrating the site of injury. In this regard, androgens have been reported to stimulate non-hair follicle keratinocyte proliferation and to enhance keratinocyte growth factor expression, suggesting that a reduction in andro- 
gen levels would potentially delay, rather than accelerate, epithelialization $(36,37)$. Our preliminary data also suggest that androgens stimulate dermal epithelial proliferative responses. Taken together, these reports suggest that the reduction in androgen levels may accelerate healing via effects on mesenchymal and/or inflammatory cells, and not by direct effects on epithelial cells at the migrating epidermal edge.

Recent studies suggest that in the absence of infection, the inflammatory response is excessive, resulting in tissue breakdown in both acute and age-related chronic wound healing states (21). A key mechanism by which gonadal androgens participate in the pathogenesis of impaired wound healing appears to involve a marked increase in the inflammatory response and upregulation of proinflammatory cytokines, including TNF- $\alpha$. Although TNF- $\alpha$ has been implicated in a variety of reproductive processes and is expressed in a number of hormonally responsive tissues $(38,39)$, no direct association between TNF- $\alpha$ and androgens in local tissue inflammation has been investigated. We observed an inflammatory response in the wounds of wild-type intact animals associated with enhanced and specific expression of tissue TNF- $\alpha$ that was dramatically curtailed in the castrated mice, suggesting that TNF- $\alpha$ is a crucial mediator of excessive inflammation in the presence of androgens. Moreover, we have demonstrated a delay in collagen deposition and healing in health status-defined elderly males compared with age-matched females. This delay correlates with increasing levels of systemic testosterone and is associated with increased tissue expression of TNF- $\alpha$. In addition, intrinsic aging in both sexes appears to correlate with increased levels of TNF- $\alpha$ in wounds. In this regard, it is interesting that several reports suggest that aging is a state of enhanced systemic inflammation, with increased circulating levels and mononuclear cell production of TNF- $\alpha$ (40-42). In aged males, this global phenomenon of cell activation appears to be exaggerated by the effects of pervasive testosterone.

Since gonadal testosterone and its precursor, androstenedione, may be aromatized in the skin to estrone $/ 17 \beta$-estradiol, with subsequent action via the estrogen receptor, we investigated the specific signaling mechanisms whereby castration modulated wound healing. Blockade of the AR with flutamide accelerated the healing response in a similar fashion to castration, being associated with a dampened inflammatory response and reduced TNF- $\alpha$ expression in vivo. These data suggested that AR mediated the inhibitory effects of gonadal steroids on tissue repair. Moreover, in vitro studies using murine macrophages confirmed that the direct upregulation of TNF- $\alpha$ expression by testosterone is inhibited by AR blockade and not by estrogen receptor inhibition. LPS induction of TNF- $\alpha$ production by macrophages, which probably occurs through NF- $\kappa B$ activation (43), is enhanced by androgen treatment and inhibited by AR blockade in a dose-dependent fashion. The upregulation of TNF- $\alpha$ appears to act as an integral component of androgen's proinflammatory effects in vivo, a pathway that may be applicable to other pathophysiological processes involving androgens, such as sepsis, protection against parasitic load, and the response to endotoxemia $(13,44,45)$. Using EMSA, Supakar et al. (46) have observed physical interaction of NF- $\kappa$ B p50 and p65 subunits with the promoter region of the AR gene in vitro. The extent to which the in vitro interactions of $A R$ and NF- $K B$ reflect physiological interactions that occur within an organism remains unknown, but the increased NF- $\kappa B$ binding activity observed in wound tissue, inhibited by AR blockade, confirms a direct or indirect effect of AR on NF- $\kappa B$ activity.

Reduced local and systemic estrogen in elderly males may contribute to age-related delayed healing. However, whereas estrogen replacement reverses the impaired healing phenotype in elderly females, this is not the case in males, suggesting alternative mechanisms inhibiting healing in males (4). Our novel data not only suggest that these results may relate to the maintenance of inhibitory androgen levels in males, but additionally implicate testosterone in the delayed repair response in males compared with females. The clinical consequences of this observation are only now being appreciated: being male is a highly significant risk factor for delayed healing in age-related chronic wound repair (5). The variability in levels of testosterone in the aged may also facilitate identification of those males who are at greatest risk of impaired healing. Such sex differences in wound healing may relate to the requirement for an estrogen-mediated rapid repair response throughout the female reproductive years, such as after menstruation and parturition. By contrast, the critical role of testicular macrophage TNF- $\alpha$ in Leydig cell testosterone secretion, coupled with androgen-induced macrophage TNF- $\alpha$ expression, suggests that a positive feedback loop between androgens and TNF- $\alpha$ that is important in reproductive functions occurs in the local cutaneous healing response. The subsequent proinflammatory picture, important for a generalized rapidfire response to local tissue infection and counteracted to a degree in youth by higher local estrogen levels, results in impaired cutaneous healing in the elderly. We suggest that systemic or local androgen blockade, possibly in synergistic combination with local estrogen therapy, may be a novel, cost-effective, and safe therapeutic strategy to accelerate healing in elderly males.

\section{Acknowledgments}

Gillian Ashcroft is a Wellcome Trust Senior Fellow in Clinical Science. ICI 182780 was kindly donated by Matt Burow, Tulane University, New Orleans, Louisiana, USA.

\footnotetext{
1. Ashcroft, G.S., et al. 1997. Age-related differences in the temporal and spatial regulation of matrix metalloproteinases (MMPs) in normal skin and acute cutaneous wounds of healthy humans. Cell Tissue Res. 290:581-591. 2. Herrick, S.E., et al. 1997. Up-regulation of elastase in acute wounds of healthy aged humans and chronic venous leg ulcers is associated with matrix degradation. Lab. Invest. 77:281-288.
} 
3. Ashcroft, G.S., Horan, M.A., and Ferguson, M.W.J. 1998. Aging alters the inflammatory and endothelial cell adhesion molecule profiles during human cutaneous wound healing. Lab. Invest. 78:47-58.

4. Ashcroft, G.S., Greenwell-Wild, T., Horan, M.A., Wahl, S.M, and Ferguson, M.W.J. 1999. Topical estrogen accelerates cutaneous wound healing in aged humans associated with an altered inflammatory response. Am. J. Pathol. 155:1137-1146.

5. Taylor, R.J., Taylor, A.D., and Smyth, J.V. 2002. Using an artificial network to predict healing times and risk factors for venous leg ulcers. J. Wound Care. 11:101-105.

6. Perry, H.M. 1999. The endocrinology of aging. Clin. Chem. 45:1369-1376

7. Labrie, F., Luu-The, V., Labrie C., and Simard, J. 2001. DHEA and its transformation into androgens and estrogens in peripheral target tissues: intracrinology. Front. Neuroendocrinol. 22:185-212.

8. Van den Beld, A.W., de Jong, F.H., Grobbee, D.E., Pols, H.A., and Lamberts, S.W. 2000. Measures of bioavailable serum testosterone and estradiol and their relationships with muscle strength, bone density, and body composition in elderly men. J. Clin. Endocrinol. Metab. 85:3276-3282

9. Ashcroft, G.S., et al. 1997. Estrogen accelerates cutaneous wound healing associated with an increase in TGF- $\beta 1$ levels. Nat. Med. 3:1209-1215.

10. Liesenfeld, O., Nguyen, T.A., Pharke, C., and Suzuki, Y. 2001. Importance of gender and sex hormones in regulation of susceptibility of the small intestine to peroral infection with Toxoplasma gondii tissue cysts. J. Parasitol. 87:1491-1493.

11. McCrohon, J.A., Jessup, W., Handelsman, D.J., and Celermajer, D.S. 1999. Androgen exposure increases human monocyte adhesion to vascular endothelium and endothelial cell expression of vascular cell adhesion molecule-1. Circulation. 99:2317-2322.

12. Angele, M.K., et al. 1998. Testosterone and/or low estradiol: normally required but harmful immunologically for males after trauma-hemorrhage. J. Trauma. 44:78-85.

13. Schroder, J., Kahlke, V., Staubach, K.H., Zabel, P., and Stuber, F. 1998 Sex differences in human sepsis. Arch. Surg. 133:1200-1205.

14. Gornstein, R.A., Lapp, C.A., Bustos-Valdes, S.M., and Zamorano, P. 1999. Androgens modulate interleukin- 6 production by gingival fibroblasts in vitro. J. Periodontol. 70:604-609.

15. Angele, M.K., et al. 1999. Sex steroids regulate pro- and anti-inflammatory cytokine release by macrophages after trauma-hemorrhage. Am.J. Physiol. 277:C35-C42.

16. Messingham, K.A., Shirazi, M., Duffner, L.A., Emanuele, M.A., and Kovacs, E.J. 2001. Testosterone receptor blockade restores cellular immunity in male mice after burn injury. J. Endocrinol. 169:299-308.

17. Hofbauer, L.C., Ten, R.M., and Khosla, S. 1999. The anti-androgen hydroxyflutamide and androgens inhibit interleukin- 6 production by an androgen-responsive human osteoblastic cell line. J. Bone Miner. Res. 14:1330-1337.

18. Padgett, D.A., and Loria, R.M. 1998. Endocrine regulation of murine macrophage function: effects of dehydroepiandrosterone, androstenediol, and androstenetriol. J. Neuroimmunol. 84:61-68.

19. Ozveri, E.S., et al. 2001. Estrogens ameliorate remote organ inflammation induced by burn injury in rats. Inflamm. Res. 50:585-591.

20. Imada, S., et al. 1997. Promoting effects and mechanisms of action of androgen in bladder carcinogenesis in male rats. Eur. Urol. 31:360-364.

21. Ashcroft, G.S., et al. 2000. Secretory leukocyte protease inhibitor mediates non-redundant functions necessary for normal wound healing. Nat. Med. 6:1147-1153

22. Li, M., et al. 1998. Nonneural nuclear inclusions of androgen receptor protein in spinal and bulbar muscular atrophy. Am. J. Pathol. 153:695-701.

23. Baratta, M., et al. 2000. Role of androgens in proliferation and differentiation of mouse mammary epithelial cell line HC11. J. Endocrinol. 167:53-60.

24. McCartney-Francis, N.L., Song, X.Y., Mizel, D.E., Wahl, C.L., and Wahl, S.M. 1999. Hemoglobin protects from streptococcal cell wall-induced arthritis Arthritis Rheum 42:1119-1127.

25. Pelletier, G. 2000. Localization of androgen and estrogen receptors in rat and primate tissues. Histol. Histopathol. 15:1261-1270.

26. Chanda, S., Robinette, C.L., Couse, J.F., and Smart, R.C. 2000. 17betaestradiol and ICI-182780 regulate the hair follicle cycle in mice through an estrogen receptor-alpha pathway. Am. J. Pbysiol. Endocrinol. Metab. 278:E202-E210.

27. Jahoda, C.A., and Reynolds, A.J. 2001. Hair follicle dermal sheath cells: unsung participants in wound healing. Lancet. 358:1445-1448.

28. Panteleyev, A.A., Botchkareva, N.V., Sundberg, J.P., Christiano, A.M., and Paus, R. 1999. The role of the hairless (hr) gene in the regulation of hair follicle catagen transformation. Am. J. Pathol. 155:159-171.

29. Song, X.Y., et al. 2000. Suppression of streptococcal cell wall-induced arthritis by human chorionic gonadotropin. Arthritis Rheum. 43:2064-2072.

30. Anderson, R.A. 1998. Follistatin and activin A production by the male reproductive tract. Hum. Reprod. 13:3319-3325.

31. Wankell, M., et al. 2001. Impaired wound healing in transgenic mice overexpressing the activin antagonist follistatin in the epidermis. EMBO J. 20:5361-5372.

32. Munz, B., et al. 1999. Overexpression of activin A in the skin of transgenic mice reveals new activities of activin in epidermal morphogenesis, dermal fibrosis and wound repair. EMBO J. 18:5205-5215.

33. Lubahn, D.B., et al. 1998. Cloning of human androgen receptor complementary DNA and localization to the X chromosome. Science. 240:327-330.

34. Chang, C.S., Kokontis, J., and Liao, S.T. 1988. Molecular cloning of human and rat complementary DNA encoding androgen receptors. Science. 240:324-326.

35. Wiren, K.M., Zhang, X., Chang, C., Keenan, E., and Orwoll, E.S. 1997. Transcriptional up-regulation of the human androgen receptor by androgen in bone cells. Endocrinology. 138:2291-2300.

36. Planz, B., Wang, Q., Kirley, S.D., Lin, C.W., and McDougal, W.S. 1998 Androgen responsiveness of stromal cells of the human prostate: regulation of cell proliferation and keratinocyte growth factor by androgen. J. Urol. 160:1850-1855.

37. Planz, B., Wang, Q., Kirley, S.D., Marburger, M., and McDougal, W.S. 2001. Regulation of keratinocyte growth factor receptor and androgen receptor in epithelial cells of the human prostate. J. Urol. 166:678-683.

38. Hutson, J.C. 1993. Secretion of tumor necrosis factor alpha by testicular macrophages. J. Reprod. Immunol. 23:63-72.

39. Warren, D.W., Pasupuleti, V., Lu, Y., Platler, B.W., and Horton, R. 1990. Tumor necrosis factor and interleukin-1 stimulate testosterone secretion in adult male rat Leydig cells in vitro. J. Androl. 11:353-360.

40. Marik, P.E., and Zaloga, G.P. 2001. The effect of aging on circulating levels of proinflammatory cytokines during septic shock. Norasept II Study Investigators. J. Am. Geriatr. Soc. 49:5-9.

41. Maes, M., et al. 1999. Inflammatory markers in younger vs elderly normal volunteers and in patients with Alzheimer's disease. J. Psychiatr. Res. 33:397-405.

42. Fagiolo, U., et al. 1992. Increased cytokine production by peripheral blood mononuclear cells from healthy elderly people. Ann. NY Acad. Sci. 663:490-493

43. Ripple, M.O., Henry, W.F., Schwarze, S.R., Wilding, G., and Weindruch, R. 1999. Effect of antioxidants on androgen-induced AP-1 and NF-kappaB DNA-binding activity in prostate carcinoma cells. J. Natl. Cancer Inst. 91:1227-1232.

44. Remoue, F., et al. 2002. Functional specific binding of testosterone to Schistosoma haematobium 28-kilodalton glutathione S-transferase. Infect. Immun. 70:601-605

45. Laubach, V.E., Foley, P.L., Shockey, K.S., Tribble, C.G., and Kron, I.L. 1998. Protective roles of nitric oxide and testosterone in endotoxemia: evidence from NOS-2-deficient mice. Am. J. Physiol. 275:H2211-H2218.

46. Supakar, P.C., and Roy, A.K. 1996. Role of transcription factors in the age-dependent regulation of the androgen receptor gene in rat liver. Biol. Signals. 5:170-179. 